\title{
Can Intraoperative Fluorescence Imaging Identify All Lesions While the Road Map Created by Preoperative Nuclear Imaging Is Masked?
}

\author{
Phillipa Meershoek ${ }^{1}$, Tessa Buckle ${ }^{1,2}$, Matthias N. van Oosterom ${ }^{1,2}$, Gijs H. KleinJan ${ }^{1,2}$, Henk G. van der Poel ${ }^{2}$, \\ and Fijs W.B. van Leeuwen ${ }^{1,2}$ \\ ${ }^{I}$ Interventional Molecular Imaging Laboratory, Department of Radiology, Leiden University Medical Center, Leiden, The \\ Netherlands; and ${ }^{2}$ Department of Urology, Netherlands Cancer Center and Antoni van Leeuwenhoek Hospital, Amsterdam, \\ The Netherlands
}

Tracers can help visualize the lymphatic drainage patterns and sentinel nodes (SNs) of individual prostate cancer patients. To determine the role of nuclear medicine in surgical guidance, in particular the positional guidance of a SPECT/CT-based 3dimensional imaging road map, in this process we studied to what extent fluorescence guidance underestimated the number of target lesions relative to radioguidance. Methods: SPECT/CT imaging was performed after intraprostatic tracer administration of either indocyanine green (ICG)- ${ }^{99 \mathrm{~m} T c-n a n o c o l l o i d ~(h y b r i d-t r a c e r ~ g r o u p) ~}$ or $99 \mathrm{mTC}$-nanocolloid to create a road map that depicted all SNs. Patients who received ${ }^{99 m}$ Tc-nanocolloid were injected with "free" ICG immediately before surgery. Before unmasking, fluorescence guidance was used for intraoperative $\mathrm{SN}$ identification. This was followed by extended pelvic lymph node dissection (ePLND). After unmasking of the SPECT/CT images, the number of missed SNs was recorded and their resection was pursued when there was no risk of intraoperative complications. Results: Preoperative SPECT/ $\mathrm{CT}$ revealed no differences in the $\mathrm{SN}$ identification rate between ICG- ${ }^{99 m}$ Tc-nanocolloid and ${ }^{99 m}$ Tc-nanocolloid. However, fluorescence guidance allowed intraoperative removal of all SNs in only $40 \%$ of patients in the hybrid-tracer group and $20 \%$ of patients in the free-ICG group. Overall, $75.9 \%$ of the intraoperatively resected SNs in the hybrid-tracer group and $51.8 \%$ of the SNs in the free-ICG group were removed solely under fluorescence guidance. During ePLND, 22 additional SNs were resected (7 in the hybrid-tracer group and 15 in the free-ICG group). After unmasking, 18 remaining SNs were identified (6 in the hybrid group and 12 in the free-ICG group). In the free-ICG group, ex vivo evaluation of the excised specimens revealed that 14 SNs removed under ePLND or after unmasking contained radioactivity but no fluorescence. Conclusion: The preoperative imaging road map provided by SPECT/CT enhanced the detection of prostate SNs in more ectopic locations in 17 of the 25 patients, and the hybrid tracer ICG-99mTc-nanocolloid was shown to outperform free ICG. Overall, fluorescenceguided pelvic nodal surgery underestimated the number of SNs in $60 \%-80 \%$ of patients.

\footnotetext{
Received Aug. 16, 2019; revision accepted Oct. 7, 2019.

For correspondence or reprints contact: Fijs W.B. van Leeuwen, Interventional Molecular Imaging Laboratory, Department of Radiology, Albinusdreef 2, 2300 RC, Leiden, The Netherlands.

E-mail: f.w.b.van_leeuwen@lumc.nl

Published online Nov. 11, 2019.

COPYRIGHT (C 2020 by the Society of Nuclear Medicine and Molecular Imaging.
}

Key Words: image-guided surgery; prostate cancer; sentinel node biopsy; SPECT/CT; robot-assisted surgery; fluorescence

J Nucl Med 2020; 61:834-841

DOI: 10.2967/jnumed.119.235234

\section{$\mathbf{I}_{\mathrm{n}}$} radioguided surgery, preoperative imaging findings support lesion identification during the intervention. The value that preoperative imaging provides is underlined by the impact that 3-dimensional imaging road maps have had on known radioguidance procedures such as sentinel node (SN) resections (SPECT/CT-based identification of aberrant tracer uptake) and PSMA-expressiontargeted resections (PET/CT- and SPECT/CT-based target definition) (1-3). The value of knowing where lesions are located during surgical procedures in relation to the patient's anatomic context is especially eminent when resections are performed in complex anatomies (e.g., the pelvic area).

The success of radioguidance during surgical procedures, fueled by calls for radioactivity-free alternatives, has sparked the revival of fluorescence-guided surgery (4). For instance, the near-infrared fluorescent dye indocyanine green (ICG) has been applied for fluorescence-based lymphangiography in prostate cancer patients $(5,6)$. The clinical growth of fluorescence-guided surgery has been further accommodated by the commercial availability of nearinfrared fluorescence cameras that depict fluorescence images in anatomic context $(7,8)$. Unfortunately, fundamental photophysical limitations such as the tissue attenuation of light limit the use of intraoperative fluorescence guidance to superficially located lesions $(<1.0 \mathrm{~cm})$, in surgically exposed tissues $(9,10)$. In patients for whom lesion locations are not accurately defined, this limitation could possibly result in underestimation of the number of lesions.

In an attempt to combine the strengths of radioguidance and fluorescence guidance and to overcome the limitation of light attenuation, in prostate cancer patients "free" ICG has been used in combination with radioguidance (preoperative imaging, such as SPECT/CT, and intraoperative $\gamma$-tracing) provided by traditional nanocolloids (e.g., ${ }^{99 \mathrm{~m} T c-n a n o c o l l o i d) ~(11) . ~ A ~ l i m i t i n g ~ f a c t o r ~ i n ~}$ the use of such a cocktail of tracers is that the individual tracers have different pharmacokinetic properties (12), which can yield a mismatch in radioactive and fluorescence findings (13). To prevent 
such a mismatch and to provide fully integrated radioguidance and fluorescence guidance toward the identical lesions, bimodal, or rather hybrid, tracers such as ICG- ${ }^{99 \mathrm{~m}} \mathrm{Tc}$-nanocolloid have been introduced $(2,14,15)$.

The clinical use of ICG- ${ }^{99 \mathrm{~m}} \mathrm{Tc}$-nanocolloid has in the past allowed us to study the concentration-dependent detection sensitivity of the individual modalities (14) but can also be used to determine to what extent fluorescence guidance underestimates the number of target lesions in a patient. This effect was studied in a masked, randomized controlled trial wherein prostate cancer patients received either ICG- ${ }^{99 \mathrm{~m}} \mathrm{Tc}$-nanocolloid or ${ }^{99 \mathrm{~m}} \mathrm{Tc}$-nanocolloid and free ICG before robot-assisted SN biopsy and extended pelvic lymph node dissection (ePLND). SN resection and ePLND were initially performed under fluorescence guidance alone, and the SNs identified on SPECT/CT became available after unmasking. A comparison of the outcomes of intraoperative fluorescence imaging, ePLND, and SPECT/CT-guided resection provided insight into the reliability of relying on fluorescence guidance during surgery.

\section{MATERIALS AND METHODS}

\section{Patients}

The study included 25 patients with histopathologically proven $\mathrm{PCa}$, clinically NOM0 on preoperative imaging, and with a risk of nodal metastases of more than 5\% according to the Briganti nomogram, who were scheduled for a robot-assisted radical prostatectomy (16). Patients participating in a clinical trial comparing 2 methods of multimodal SN tracing were asked to participate in a substudy on the value of preoperative SPECT/CT (trial M13PSN). Patients were randomly allocated to receive either a preoperative injection into the prostate of the hybrid tracer ICG-99m Tc-nanocolloid (15 patients) or a preoperative injection of ${ }^{99 \mathrm{~m}} \mathrm{Tc}-$ nanocolloid followed by an intraoperative injection of free ICG (10 patients). The exclusion criteria were a history of iodine allergy, hyperthyroid or thyroidal adenoma, kidney insufficiency, surgical inaccessibility, or incorrect implementation of the procedure.

All procedures were in accordance with the 1964 Helsinki Declaration and its later amendments or comparable ethical standards and were performed after approval was obtained by the local ethics committee of The Netherlands Cancer Institute-Antoni van Leeuwenhoek Hospital (approval NL46580.031.13). Written informed consent was obtained from all patients before inclusion in the study.

\section{Tracer Administration and Preoperative Imaging}

The radiotracers were injected either as a hybrid tracer (ICG- ${ }^{99 m}$ Tc-nanocolloid) $(500 \mu \mathrm{g}$ of human serum albumin in 2 $\mathrm{mL}$ ) or as a monomodal tracer ( ${ }^{99 \mathrm{~m}} \mathrm{Tc}$-nanocolloid) with a second injection of free ICG into the prostate minutes before the ePLND. ICG- ${ }^{99} \mathrm{~m}$ Tc-nanocolloid contained $0,25 \mathrm{mg}$ of ICG (total volume), whereas a free-ICG injection meant $5 \mathrm{mg}$ of ICG were administered (20-fold more) to the patient, also constituting a 2-fold increase in injected volume ( $4 \mathrm{~mL}$ total). No difference in the amount of radioactivity used could be detected between the 2 groups (administered doses of $205.1 \pm 17.4 \mathrm{MBq}$ and $207.0 \pm 15.3 \mathrm{MBq}$, respectively, $P=0.793)$.

After radiotracer administration, lymphatic mapping, including SPECT/ $\mathrm{CT}$, was performed as previously described $(15,17)$. On the basis of the reproducibility of lymphatic drainage for ${ }^{99 \mathrm{~m}} \mathrm{Tc}-$ nanocolloid and ICG-99mTc-nanocolloid (17), SNs identified on SPECT/CT were considered to be the gold standard.
Patients in the free-ICG group received an additional 4 transrectal intraprostatic injections of ICG 5-10 min before initiation of the procedure, while under full anesthesia.

\section{Surgical Procedure}

Approximately $4 \mathrm{~h}$ after preoperative injection of the hybrid or radioactive tracer, patients were transferred to the operation room. All procedures were performed by 2 experienced robotic surgeons using a da Vinci Si surgical system (Intuitive Surgical). Initially, the operating surgeon was masked to the SPECT/CT imaging data and pursued the SNs intraoperatively with near-infrared fluorescence imaging only. This was done using the ICG-tuned Firefly camera integrated in the da Vinci Si surgical system. Fluorescence-based SN resection was followed by resection of the extended pelvic lymph node template (ePLND) (obturator fossa and external iliac vessels, both up to the ureter vessel crossing on both sides). To identify ${ }^{99 \mathrm{~m}} \mathrm{Tc}-$ and ICG-containing nodes ex vivo, additional measurements were performed on the surgical specimens using a modified PDE-mod near-infrared fluorescence camera system (Hamamatsu Photonics (7)) and a $\gamma$-probe (Neoprobe; Johnson and Johnson Medical). SNs removed in the ePLND template but not primarily detected by fluorescence guidance intraoperatively were labeled $\mathrm{SN}$ when containing an ex vivo $\gamma$-signal above background level.

Subsequently, the surgeon was unmasked to the preoperative SPECT/CT images. These images were then consulted to confirm removal of all SNs under fluorescence guidance and or during ePLND. SNs that had been missed were pursued with the positional guidance of SPECT/CT. In vivo detection using an intraoperative $\gamma$-probe was used only after unmasking. Excised specimens were evaluated ex vivo to validate their fluorescent and radioactive content.

\section{Quantification Lesion Underestimation Under \\ Fluorescence Guidance}

The number and location of the SNs identified using fluorescence guidance alone, both within and outside the ePLND template, were noted for the 2 groups. After unmasking, the number and location of the intraoperatively identified SNs were compared with the number and location of SNs identified on preoperative imaging. Correlation between the radioactive and fluorescent content of the excised specimens was included to identify SNs that were radioactive but not fluorescent.

\section{Pathology}

The excised lymph nodes were pathologically examined for the presence of macro- or micrometastases or isolated tumor cells, according to previously described protocols $(8,18)$. The resected prostatectomy specimens were examined and classified for pathologic tumor stage $(8)$.

\section{Complications}

Intraoperative and postoperative complications were scored. Postoperative complications were included when occurring within $90 \mathrm{~d}$ after surgery and were divided according to the Clavien Dindo (CD) score.

\section{Follow-up}

Biochemical recurrence was defined as a PSA level of at least 0.2 $\mathrm{ng} / \mathrm{mL}$ after prostatectomy and ePLND. Patients were screened (watchand-wait principle) for biochemical recurrence every 6 mo postoperatively for up to 57 mo after surgery. No adjuvant radiotherapy or androgen ablation therapy was used in the absence of biochemical recurrence. When PSA levels over $0.2 \mathrm{ng} / \mathrm{mL}$ were detected after prostatectomy, ${ }^{68} \mathrm{Ga}$-PSMA PET imaging was performed to detect the location of disease recurrence. 


\section{Statistics}

Statistical analysis was performed using SPSS, version 22 (IBM). $\chi^{2}$ and Fisher exact tests were used to compare the distributions of patient characteristics. The endpoint of the study was the number of patients with missed SNs apparent on unmasking of the SPECT/CT results. Use of the hybrid tracer was considered necessary when in at least 1 of 25 men additional nodes were found after unmasking.

\section{RESULTS}

\section{Patients}

Patient characteristics are summarized in Table 1. All patients had cancer with a clinical Gleason score of 7 or higher before surgery, and the clinical stages ranged between cT1c and cT3b.
The mean Briganti risk score for nodal metastases was $16.6 \%$ $(13 \%)$. No significant differences were seen between patients in the hybrid-tracer and free-ICG groups.

\section{Preoperative Imaging Findings}

In total, $111 \mathrm{SNs}$ were identified on preoperative SPECT/CT (Table 2), with a mean of $4.4 \pm 2.5$ SNs identified per patient in the complete patient group. Of these SNs, 57 (3.8 \pm 1.8 per patient) were in the hybrid-tracer group and 54 (5.4 \pm 3.0 per patient) were in the free-ICG group. Of the $82 \mathrm{SNs}$ identified within the eLNPD template, 50 were in the hybrid-tracer group (87.7\% of SNs within this group) and $32(59.3 \%$ of SNs within this group) were in the free-ICG group. Overall, the most prevalent nodal location was the obturator fossa $(31.5 \%$ of all SNs; Table 2).

TABLE 1

Patient Characteristics

\begin{tabular}{|c|c|c|c|c|}
\hline Characteristic & $\begin{array}{l}\text { All patients } \\
(n=25)\end{array}$ & $\begin{array}{l}\text { Hybrid-tracer } \\
\text { group }(n=15)\end{array}$ & $\begin{array}{c}\text { Free-ICG } \\
\text { group }(n=10)\end{array}$ & $P$ \\
\hline Age at surgery $(y)$ & $65.4(6.1)$ & $64.3(8.4)$ & $67.4(4.4)$ & 0.247 \\
\hline PSA at diagnosis & $8.6(3.9)$ & $9.1(4.6)$ & $7.7(2.3)$ & 0.383 \\
\hline PSA range at diagnosis $(\mathrm{ng} / \mathrm{mL})$ & $2.7-22.5$ & $2.7-22.5$ & $4.31-12.04$ & \\
\hline Clinical stage & & & & 0.870 \\
\hline cT1c & 5 & 4 & 1 & \\
\hline cT2a & 4 & 2 & 2 & \\
\hline $\mathrm{cT} 2 \mathrm{~b}$ & 3 & 1 & 2 & \\
\hline cT2c & 7 & 4 & 3 & \\
\hline сT3a & 5 & 3 & 2 & \\
\hline cT3b & 1 & 1 & 0 & \\
\hline Biopsy Gleason sum & & & & 1.000 \\
\hline 6 & 2 & 1 & 1 & \\
\hline 7 & 17 & 10 & 7 & \\
\hline 8 & 3 & 2 & 1 & \\
\hline 9 & 3 & 2 & 1 & \\
\hline Prostate volume (mL) & $50.2(21)$ & $48.1(20.0)$ & $52.4(25.8)$ & 0.641 \\
\hline Briganti score & $16.6 \%(16.1)$ & $16.6(15.6)$ & $16.6(17.8)$ & 0.996 \\
\hline Pathologic stage & & & & 0.596 \\
\hline pT2a & 4 & 1 & 3 & \\
\hline pT2c & 14 & 9 & 5 & \\
\hline pT3a & 5 & 3 & 2 & \\
\hline pT3b & 1 & 1 & 0 & \\
\hline pT4 & 1 & 1 & 0 & \\
\hline Pathologic Gleason sum & & & & 0.194 \\
\hline 6 & 3 & 3 & 0 & \\
\hline 7 & 15 & 7 & 8 & \\
\hline 8 & 3 & 3 & 0 & \\
\hline 9 & 2 & 2 & 2 & \\
\hline Nodal status & & & & 1.00 \\
\hline pNO & 19 & 11 & 8 & \\
\hline pN1 & 6 & 4 & 2 & \\
\hline
\end{tabular}

Qualitative data are expressed as numbers; continuous data are expressed as mean followed by SD in parentheses. 
TABLE 2

Results for Identified and Removed SNs and LNs: SPECT/CT and Ex Vivo Imaging

\begin{tabular}{|c|c|c|c|}
\hline Parameter & $\begin{array}{l}\text { All patients } \\
(\mathrm{n}=25)\end{array}$ & $\begin{array}{l}\text { Hybrid-tracer } \\
\text { group }(\mathrm{n}=15)\end{array}$ & $\begin{array}{c}\text { Free-ICG } \\
\text { group }(n=10)\end{array}$ \\
\hline \multicolumn{4}{|l|}{ SPECT/CT } \\
\hline Total identified SNs & 111 & 57 & 54 \\
\hline Mean SNs per patient & $4.4(S D, 2.5)$ & $3.8(\mathrm{SD}, 1.8)$ & $5.4(\mathrm{SD}, 3.0)$ \\
\hline SNs inside ePLND template & $82(73.9 \%)$ & $50(87.7 \%)$ & $32(59.3 \%)$ \\
\hline External iliac & 26 & 16 & 10 \\
\hline Internal iliac & 21 & 13 & 8 \\
\hline Obturator fossa & 35 & 21 & 14 \\
\hline SNs outside ePLND template & $29(26.1 \%)$ & $7(12.3 \%)$ & $22(40.7 \%)$ \\
\hline Common iliac & 12 & 4 & 8 \\
\hline Paravesical & 3 & 3 & 0 \\
\hline Presacral & 5 & 0 & 5 \\
\hline Pararectal & 6 & 0 & 6 \\
\hline Inguinal & 3 & 0 & 3 \\
\hline
\end{tabular}

Data are numbers.

\section{Intraoperative Findings}

Intraoperatively, the hybrid-tracer group allowed visualization of the exact same lesions as identified on preoperative SPECT, whereas the literature indicates there could be discrepancy between SNs detected with ${ }^{99 \mathrm{~m}} \mathrm{Tc}$-nanocolloid and those detected with free ICG (13). In total, 114 SNs were resected (Table 3; Fig. 1). The fact that fluorescence imaging was able to discriminate between cluster nodes that were seen as a single hotspot on SPECT/CT helps explain why the number was slightly higher than the SNs defined at preoperative imaging (19). In 8 patients $(6 / 15$ [40\%] in the hybrid-tracer group and $2 / 10$ [20\%] in the free-ICG group), all SNs could be removed under fluorescence guidance alone. In the remaining 17 patients, one or more SNs were missed. Overall, $75.9 \%$ of the SNs in the hybrid-tracer group (44/58) were removed under fluorescence guidance, compared with $51.8 \%$ (29/ 56 ) in the free-ICG group (Table 3). In addition, $24 \%$ more fluorescent non-SNs were removed in the free-ICG group than in the hybrid-tracer group (13 non-SNs [62\%] vs. 8 non-SNs [38\%], respectively).

Of the 58 fluorescent $\mathrm{SNs}$ found in the hybrid-tracer group, $75.9 \%$ (44 SNs) were located within the ePLND template (Table 3; Fig. 1). In agreement with the preoperative findings, only $51.8 \%$ (29 SNs) of the 56 fluorescent SNs in the free-ICG group were located within the ePLND template. This finding underlines the difference in drainage kinetic between free ICG and radiocolloids (12).

After unmasking of the SPECT/CT images to the surgeon, 18 SNs (in 11 patients) were identified that had not yet been resected (6 SNs [10.3\%] in the hybrid-tracer group and 12 SNs [21.4\%] in the free-ICG group [Table 3; Fig. 1]). Of these initially missed SNs, only 1 was located within the ePLND template (external iliac region; Fig. 2); the remaining nodes were located outside the ePLND template (Table 3). To avoid damage to healthy tissue, only the SN located within the ePLND template was surgically removed.

Ex vivo evaluation of the radioactive and fluorescent content of the excised specimens revealed that of the 27 SNs that had been removed under ePLND or after unmasking of the preoperative
SPECT images in the free-ICG group, 14 (in 7/10 [70\%] of patients) contained radioactivity but no fluorescence (Table 2; Fig. 1). This mismatch was not seen in the hybrid-tracer group.

\section{Pathology}

Pathologic analysis yielded nonradical resection margins after prostatectomy in 8 patients and $\mathrm{LN}$ metastases in 6 patients $(24 \%$; Table 4). Of the 13 metastases found, 10 were identified in nodes specified as SNs on preoperative imaging. Nine of these SNs were in the hybrid-tracer group, and 1 was in the free-ICG group (Fig. 1). In 4 patients $(66.7 \%)$, more than 1 (range, 2-4) positive node was found. In 3 of these patients, additional metastases were found in non-SN nodes (1 per patient). In 1 patient in the free-ICG group, a false-negative but tumor-positive SN was found in the ePLND specimens but was missed on SPECT/CT; the preoperatively specified SNs and fluorescent nodes in this patient were all tumor-negative. Although displaying 1,600 counts with the $\gamma$-probe ex vivo, this $\mathrm{SN}$ was not classified as an $\mathrm{SN}$ on preoperative imaging. This finding indicates that in some cases (1/111 SNs; $1 \%)$ the sensitivity of SPECT/CT may limit nodal identification.

\section{Complications}

The overall complication rate within $90 \mathrm{~d}$ after surgery was $16.0 \%$, which is in line with previously reported complication rates (20). No intraoperative complications were recorded, and no substantial difference in postsurgical complications was seen between the hybrid-tracer group and the free-ICG group; in the hybrid-tracer group, $1 \mathrm{CD}$ type 2 complication (pulmonary embolism) and $1 \mathrm{CD}$ type $3 \mathrm{~b}$ complication (cicatricial port site hernia) were registered. In the free-ICG group, $1 \mathrm{CD}$ type 1 complication (incontinence) and $1 \mathrm{CD}$ type 2 complication (urinary tract infection) were recorded.

\section{Follow-up}

A mean follow-up of 23.3 mo (range, 7-57 mo) was available for 23 patients (13 in the hybrid-tracer group and 10 in the freeICG group). Of the patients with a follow-up of less than 12 mo 
TABLE 3

Results for Identified and Removed SNs and LNs: Surgery

\begin{tabular}{|c|c|c|c|}
\hline Parameter & $\begin{array}{l}\text { All patients } \\
(n=25)\end{array}$ & $\begin{array}{l}\text { Hybrid-tracer } \\
\text { group }(n=15)\end{array}$ & $\begin{array}{c}\text { Free-ICG } \\
\text { group }(n=10)\end{array}$ \\
\hline Resected SNs & 114 & 58 & 56 \\
\hline $\begin{array}{l}\text { SNs removed under } \\
\text { fluorescence guidance }\end{array}$ & $73(64.0 \%)$ & $44(75.9 \%)$ & $29(51.8 \%)$ \\
\hline SNs per patient & $4.6(\mathrm{SD}, 3.0)$ & $3.8(\mathrm{SD}, 2.0)$ & $5.6(\mathrm{SD}, 3.8)$ \\
\hline $\begin{array}{l}\text { Fluorescent SNs removed } \\
\text { inside ePLND template }\end{array}$ & $56(79.5 \%)$ & $34(77.3 \%)$ & $22(75.9 \%)$ \\
\hline Externa iliac & 20 & 12 & 8 \\
\hline Internal iliac & 11 & 7 & 4 \\
\hline Obturator fossa & 25 & 15 & 10 \\
\hline $\begin{array}{l}\text { Fluorescent SNs removed } \\
\text { outside ePLND template }\end{array}$ & $17(23.3 \%)$ & $10(29.4 \%)$ & $7(24.1 \%)$ \\
\hline Common iliac & 2 & 2 & 0 \\
\hline Marcille & 0 & 0 & 0 \\
\hline Cloquet & 0 & 0 & 0 \\
\hline Pararectal & 5 & 0 & 5 \\
\hline Presacral & 2 & 0 & 2 \\
\hline Umbilical ligament & 8 & 8 & 0 \\
\hline Aorta bifurcation & 0 & 0 & 0 \\
\hline Undefined & 0 & 0 & 0 \\
\hline SNs resected during eLNPD & $22(19.3 \%)$ & $7(12.1 \%)$ & $15(26.8 \%)$ \\
\hline SNs identified after unmasking of SPECT & $18(15.8 \%)$ & $6(10.3 \%)$ & $12(21.4 \%)$ \\
\hline SNs removed after unmasking & 1 & - & 1 \\
\hline SNs not removed after unmasking & 17 & 7 & 10 \\
\hline \multicolumn{4}{|l|}{ Location } \\
\hline Inside ePLND template (internal iliac) & 3 & 2 & 1 \\
\hline \multicolumn{4}{|l|}{ Outside ePLND template } \\
\hline Common iliac & 4 & 3 & 1 \\
\hline Pararectal & 8 & 2 & 6 \\
\hline Inguinal & 2 & - & 2 \\
\hline $\begin{array}{l}\text { Non-SNs removed under fluorescence } \\
\text { guidance }\end{array}$ & 21 & 8 & 13 \\
\hline \multicolumn{4}{|l|}{ Ex vivo imaging } \\
\hline $\begin{array}{l}\text { SNs that were fluorescent but } \\
\text { not radioactive }\end{array}$ & 14 & 0 & 14 \\
\hline Patients & $7(28 \%)$ & $0(0 \%)$ & $7(70 \%)$ \\
\hline
\end{tabular}

$(n=10), 90 \%$ had PSA levels of less than $0.01 \mathrm{ng} / \mathrm{mL}$. No differences in biochemical recurrence were seen between the hybridtracer group and the free-ICG group.

Of the 8 patients whose resection margins were deemed to be nonradical at pathology, 4 had PSA levels of less than $0.01 \mathrm{ng} / \mathrm{mL}$ (mean follow-up, $7.2 \mathrm{mo}$; range, 7-8 mo) and 4 had PSA levels of 0.03-7.56 ng/mL (mean follow-up, $49 \mathrm{mo}$; range, 48-50 mo). Three of the 4 patients with a biochemical recurrence had nodal metastasis. All 4 underwent additional radiotherapy. One patient $(\mathrm{PSA}<0.01 \mathrm{ng} / \mathrm{mL}$ ) died of lung carcinoma within a year after prostate cancer surgery.
The 6 patients for whom lymph node metastases were found were subjected to watch-and-wait follow-up (mean follow-up, 47,7 mo; range to date, 33-57 mo). Four received additional therapies after surgery (radiotherapy $[n=4]$ and further LN dissection of the pelvis $[n=1])$, additional chemotherapy $(n=2)$ after a rise in PSA level, and subsequent ${ }^{68} \mathrm{Ga}$-PSMA PET imaging.

\section{DISCUSSION}

Procedural cost savings would warrant simplification of imageguided surgery approaches. Hence, insight into the value of 


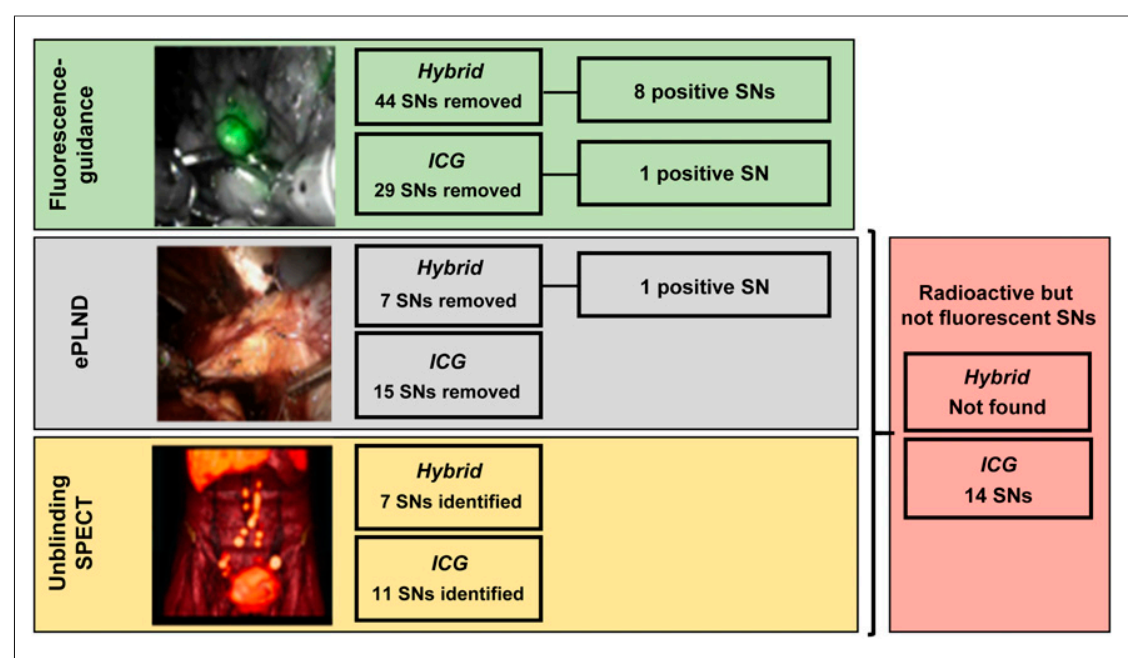

FIGURE 1. Overview of intraoperative SN removal and tumor-positive SNs.

fluorescence guidance and omission of the use of relatively costly road maps based on preoperative imaging findings is in demand. By demonstrating that masking surgeons to SPECT/CT data underestimates the number of SNs using fluorescence guidance alone, our study helps elucidate to what extent fluorescence light attenuation influences the process of image-guided surgery.

In conformation with a previous reproducibility study by Brouwer et al., no difference in the overall preoperative $\mathrm{SN}$ identification rate (radioactivity-based) was seen between ICG-99m Tc-nanocolloid and ${ }^{99 m}$ Tc-nanocolloid (17). To our surprise, the 2 groups-although patient selection was identical and patient inclusion was randomized-SPECT/CT yielded different numbers of SNs beyond the ePLND template. When comparing the intraoperative SN iden-

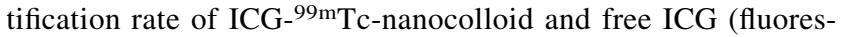
cence-based), we saw clear differences; $24.1 \%$ of SNs the hybrid-tracer group and $49.2 \%$ of SNs in the free-ICG group were missed when resection was pursued with fluorescence guidance alone. Moreover, ex vivo assessment of excised specimens in the free-ICG group revealed a mismatch between the radioactive and fluorescent content of the SNs in $70 \%$ of patients. This finding indicates a discrepancy in the distribution pattern between ${ }^{99 \mathrm{~m}} \mathrm{Tc}-$ nanocolloid and free ICG and supports the arguments behind using a hybrid tracer for image guidance rather than relying on a combination of a radioactive and fluorescent tracers. Important to note

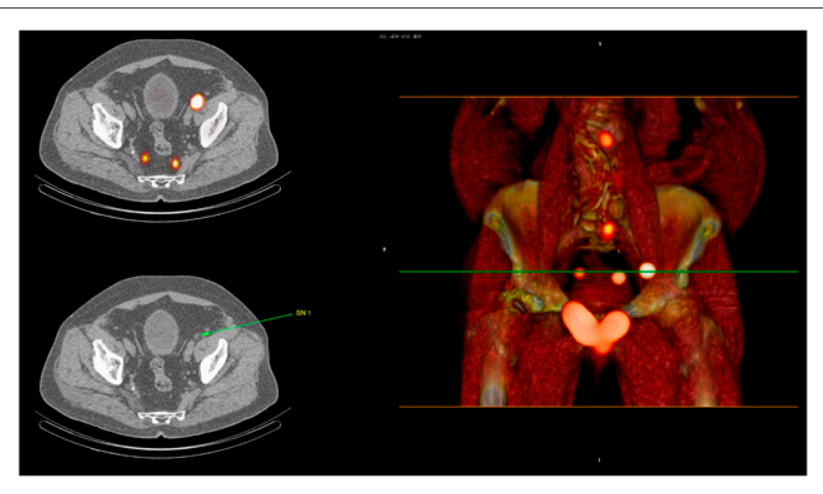

FIGURE 2. Location of iliac SN resected after unmasking of surgeon to SPECT/CT. is that the 20-times-higher dose of ICG in the free-ICG group did not lead to increased intensities that subsequently led to better in vivo $\mathrm{SN}$ identification; rather, a substantial higher number $(24 \%)$ of fluorescent non-SNs were stained and removed in the free-ICG group. These non-SNs could be considered false-positives.

The findings presented here indicate that, similar to what has been reported for radioguided surgery $(2,7,21)$, the accuracy of fluorescence-guided surgery regarding lesions in unknown locations is related to the availability of accurate surgical road maps in the form of preoperative imaging data. This indication not only supports the concept of using hybrid tracers for surgical guidance (22) but also strengthens the suggested use of intraoperative augmented and virtual-reality-based navigation to improve the position of the fluorescence camera relative to the lesion location (23).

In prostate cancer, lymphatic metastases often occur in unexpected locations (24). In line with these reports, a substantial number of SNs were found outside the ePLND template (17/73 SNs [23.3\%] in 8/25 patients $[32 \%])$. The fact that fluorescence guidance structurally misses lesions in unexpected locations means that receptor-targeted fluorescence-guided surgery performed without accompanying preoperative imaging data could translate to a poor oncologic outcome. Assuming that the use of tracers in image-guided surgery has the purpose of enabling radical surgical excision of lesions, this paints an alarming picture of the future of the field. Follow-up data from large, randomized studies that apply tumor-specific tracers will be needed to confirm if this is indeed the case. Given the superior detection sensitivity of radiotracers, this potential risk can be easily mitigated through the implementation of corresponding preoperative imaging data through the use of hybrid tracers, an exponentially growing field of research. Current efforts in hybrid-tracer developments include, for example, Cerenkov imaging (25), small molecules (26), peptides (27), mAbs (28), and nanoparticles (29).

A strength of this study was the possibility of removing the SNs that were missed under fluorescence guidance alone after unmasking of the surgeon to the preoperative SPECT images. The additional value of SPECT/CT imaging was evident despite the small sample size and was relevant for both tracer formulations, strengthening the observation. However, this setup does not allow evaluation of the complication rate or possible differences in follow-up based solely on intraoperative fluorescence guidance results. Also, the small sample size complicates a head-to-head comparison of the 2 different tracer formulations used-an issue that is currently being addressed in a follow-up study with a larger sample size.

In prostate cancer, the $\mathrm{SN}$ procedure is applied to identify micrometastases. In so doing, the procedure positively impacts recurrence values. Recently, this concept proved to be of value in PSMA PET-negative patients (30). Because not all SNs will contain metastases, the operating surgeon always has to balance the benefit of the resection against the potential harm to the patient (e.g., lymphedema, thromboembolic events, ureteral injury, and neurovascular injuries). In this study, that balancing meant that some SNs (14.9\%) were not surgically resected. Compared with indications that would have relied on receptor-targeted tracers for 
TABLE 4

Results for Identified and Removed SNs and LNs: Pathology

\begin{tabular}{|c|c|c|c|}
\hline Parameter & $\begin{array}{l}\text { All patients } \\
(n=25)\end{array}$ & $\begin{array}{l}\text { Hybrid-tracer } \\
\text { group } \\
(n=15)\end{array}$ & $\begin{array}{l}\text { Free-ICG } \\
\text { group } \\
(n=10)\end{array}$ \\
\hline \multicolumn{4}{|l|}{$\begin{array}{l}\text { Tumor-positive } \\
\text { SNs }\end{array}$} \\
\hline Nodes & 10 & 9 & 1 \\
\hline Patients & 6 & 4 & 1 \\
\hline Non-SNs removed & 320 & 177 & 143 \\
\hline \multicolumn{4}{|l|}{$\begin{array}{c}\text { Tumor-positive } \\
\text { nodes in } \\
\text { non-SNs }\end{array}$} \\
\hline Nodes & 3 & 2 & 1 \\
\hline Patients & 3 & 2 & 1 \\
\hline \multicolumn{4}{|l|}{$\begin{array}{l}\text { Tumor-positive } \\
\text { nodes inside } \\
\text { ePLND template }\end{array}$} \\
\hline External iliac & 5 & $5^{\star}$ & \\
\hline Internal iliac & 2 & 2 & \\
\hline Obturator fossa & 3 & 2 & $1^{\dagger}$ \\
\hline \multicolumn{4}{|l|}{$\begin{array}{l}\text { Tumor-positive } \\
\text { nodes outside } \\
\text { ePLND template }\end{array}$} \\
\hline Marcille & 1 & & 1 \\
\hline Cloquet & 1 & 1 & \\
\hline Pararectal & 1 & 1 & \\
\hline $\begin{array}{l}{ }^{*} 2 \text { non-SNs. } \\
{ }^{\dagger} 1 \text { non-SN. } \\
\text { Data are numbers. }\end{array}$ & & & \\
\hline
\end{tabular}

surgical guidance, using SN procedures to study the accuracy of fluorescence guidance limits the negative impact that miss rates could have had on the final patient outcome.

\section{CONCLUSION}

Intraoperative comparison between the hybrid tracer ICG-99m Tc-nanocolloid and free ICG revealed that accurate surgical guidance to ectopic SNs could not be achieved when solely relying on fluorescence guidance in 17 of the 25 patients included in this study. The preoperative imaging road map provided by SPECT/CT enhanced the detection of prostate SNs in more ectopic locations, and the hybrid tracer ICG-99m Tc-nanocolloid was shown to outperform free ICG.

\section{DISCLOSURE}

This research was supported by an NWO-STW-VICI grant (STW TTW 16141). No other potential conflict of interest relevant to this article was reported.

\section{ACKNOWLEDGMENTS}

We thank all participating patients, the entire surgical staff, and the departments of pathology and nuclear medicine of the Netherlands Cancer Institute-Antoni van Leeuwenhoek Hospital for their contribution. We also thank Nynke van den Berg for her help in archiving the findings in the database.

\section{KEY POINTS}

QUESTION: Can fluorescence-guided surgery help identify all lesions in unknown locations, or is the integrated use of a road map created by preoperative imaging mandatory?

PERTINENT FINDINGS: In a cohort study evaluating the extent to which fluorescence guidance underestimated the number of target lesions in 25 prostate cancer patients, the preoperative imaging road map provided by SPECT/CT was shown to enhance the detection of prostate SNs in more ectopic locations. The hybrid tracer ICG-99mTc-nanocolloid was shown to outperform free ICG.

IMPLICATIONS FOR PATIENT CARE: Accurate surgical guidance to ectopic SNs benefits from a hybrid approach wherein fluorescence guidance is complemented by a preoperative imaging road map provided by SPECT/CT.

\section{REFERENCES}

1. Robu S, Schottelius M, Eiber M, et al. Preclinical evaluation and first patient application of ${ }^{99 \mathrm{~m} T c-P S M A-I \& S}$ for SPECT imaging and radioguided surgery in prostate cancer. J Nucl Med. 2017;58:235-242.

2. van Leeuwen FWB, Winter A, van Der Poel HG, et al. Technologies for imageguided surgery for managing lymphatic metastases in prostate cancer. Nat Rev Urol. 2019;16:159-171.

3. Vermeeren L, van der Ploeg IM, Olmos RA, et al. SPECT/CT for preoperative sentinel node localization. J Surg Oncol. 2010;101:184-190.

4. van Leeuwen FW, Hardwick JC, van Erkel AR. Luminescence-based imaging approaches in the field of interventional molecular imaging. Radiology. 2015;276: 12-29.

5. Manny TB, Patel M, Hemal AK. Fluorescence-enhanced robotic radical prostatectomy using real-time lymphangiography and tissue marking with percutaneous injection of unconjugated indocyanine green: the initial clinical experience in 50 patients. Eur Urol. 2014;65:1162-1168.

6. Wit EM, Acar C, Grivas N, et al. Sentinel node procedure in prostate cancer: a systematic review to assess diagnostic accuracy. Eur Urol. 2017;71:596-605.

7. van den Berg NS, Miwa M, KleinJan GH, et al. (Near-infrared) fluorescenceguided surgery under ambient light conditions: a next step to embedment of the technology in clinical routine. Ann Surg Oncol. 2016;23:2586-2595.

8. KleinJan GH, van den Berg NS, Brouwer OR, et al. Optimisation of fluorescence guidance during robot-assisted laparoscopic sentinel node biopsy for prostate cancer. Eur Urol. 2014;66:991-998.

9. Stammes MA, Bugby SL, Porta T, et al. Modalities for image- and molecularguided cancer surgery. Br J Surg. 2018;105:e69-e83.

10. Teraphongphom N, Kong CS, Warram JM, Rosenthal EL. Specimen mapping in head and neck cancer using fluorescence imaging. Laryngoscope Investig Otolaryngol. 2017;2:447-452.

11. Jeschke S, Lusuardi L, Myatt A, et al. Visualisation of the lymph node pathway in real time by laparoscopic radioisotope- and fluorescence-guided sentinel lymph node dissection in prostate cancer staging. Urology. 2012;80:1080-1086.

12. Van Den Berg NS, Buckle T, Kleinjan GI, et al. Hybrid tracers for sentinel node biopsy. Q J Nucl Med Mol Imaging. 2014;58:193-206.

13. Soergel P, Kirschke J, Klapdor R, et al. Sentinel lymphadenectomy in cervical cancer using near infrared fluorescence from indocyanine green combined with technetium-99m-nanocolloid. Lasers Surg Med. 2018;50:994-1001.

14. KleinJan GH, Bunschoten A, van den Berg NS, et al. Fluorescence guided surgery and tracer-dose, fact or fiction? Eur J Nucl Med Mol Imaging. 2016;43:1857-1867.

15. van der Poel HG, Buckle T, Brouwer OR, Valdes Olmos RA, van Leeuwen FW. Intraoperative laparoscopic fluorescence guidance to the sentinel lymph node in prostate cancer patients: clinical proof of concept of an integrated functional imaging approach using a multimodal tracer. Eur Urol. 2011;60:826-833.

16. Briganti A, Larcher A, Abdollah F, et al. Updated nomogram predicting lymph node invasion in patients with prostate cancer undergoing extended pelvic lymph node dissection: the essential importance of percentage of positive cores. Eur Urol. 2012;61:480-487. 
17. Brouwer OR, Buckle T, Vermeeren L, et al. Comparing the hybrid fluorescentradioactive tracer indocyanine green- ${ }^{99 \mathrm{~m}} \mathrm{Tc}$-nanocolloid with ${ }^{99 \mathrm{~m}} \mathrm{Tc}$-nanocolloid for sentinel node identification: a validation study using lymphoscintigraphy and SPECT/CT. J Nucl Med. 2012;53:1034-1040.

18. KleinJan GH, van den Berg NS, de Jong J, et al. Multimodal hybrid imaging agents for sentinel node mapping as a means to (re)connect nuclear medicine to advances made in robot-assisted surgery. Eur J Nucl Med Mol Imaging. 2016;43:1278-1287.

19. KleinJan GH, van Werkhoven E, van den Berg NS, et al. The best of both worlds: a hybrid approach for optimal pre- and intraoperative identification of sentinel lymph nodes. Eur J Nucl Med Mol Imaging. 2018;45:1915-1925.

20. Liss MA, Palazzi K, Stroup SP, et al. Outcomes and complications of pelvic lymph node dissection during robotic-assisted radical prostatectomy. World J Urol. 2013;31:481-488.

21. van Leeuwen FW, Valdes-Olmos R, Buckle T, Vidal-Sicart S. Hybrid surgical guidance based on the integration of radionuclear and optical technologies. $\mathrm{Br}$ J Radiol. 2016;89:20150797.

22. Bugby SL, Lees JE, Perkins AC. Hybrid intraoperative imaging techniques in radioguided surgery: present clinical applications and future outlook. Clin Transl Imaging. 2017;5:323-341.

23. Van Oosterom MN, Rietbergen DDD, Welling MM, et al. Recent advances in nuclear and hybrid detection modalities for image-guided surgery. Expert Rev Med Devices. 2019;16:711-734.
24. Van den Bergh L, Joniau S, Haustermans K, et al. Reliability of sentinel node procedure for lymph node staging in prostate cancer patients at high risk for lymph node involvement. Acta Oncol. 2015;54:896-902.

25. Tamura R, Pratt EC, Grimm J. Innovations in nuclear imaging instrumentation: Cerenkov imaging. Semin Nucl Med. 2018;48:359-366.

26. Hensbergen AWB, Buckle T, van Willigen DM, et al. Hybrid tracers based on cyanine backbones targeting prostate-specific membrane antigen: tuning pharmacokinetic properties and exploring dye-protein interaction. J Nucl Med. September 3, 2019 [Epub ahead of print].

27. Kuil J, Velders AH, van Leeuwen FW. Multimodal tumor-targeting peptides functionalized with both a radio- and a fluorescent label. Bioconjug Chem. 2010;21: 1709-1719.

28. Lütje S, Heskamp S, Franssen GM, et al. Development and characterization of a theranostic multimodal anti-PSMA targeting agent for imaging, surgical guidance, and targeted photodynamic therapy of PSMA-expressing tumors. Theranostics. 2019;9:2924-2938.

29. Smith BR, Gambhir SS. Nanomaterials for in vivo imaging. Chem Rev. 2017; 117:901-986.

30. Hinsenveld FJW, Wit EMK, van Leeuwen PJ, et al. Prostate-specific membrane antigen positron emission tomography/computed tomography combined with sentinel node biopsy for primary lymph node staging in prostate cancer. $\mathrm{J} \mathrm{Nucl}$ Med. September 27, 2019 [Epub ahead of print]. 